\title{
A Dynamic Field Model of Ordinal and Timing Properties of Sequential Events
}

\author{
Flora Ferreira $^{1, \star}$, Wolfram Erlhagen ${ }^{1,3}$, and Estela Bicho ${ }^{2}$ \\ 1 Dept. Mathematics, University of Minho, 4800-058 Guimarães, Portugal \\ fjferreira@dei.uminho.pt, wolfram.erlhagen@math.uminho.pt \\ 2 Dept. Industrial Electronics, University of Minho, 4800-058 Guimarães, Portugal \\ estela.bicho@dei.uminho.pt \\ 3 Donders Institute, BCB, Radboud University Nijmegen, The Netherlands
}

\begin{abstract}
Recent evidence suggests that the neural mechanisms underlying memory for serial order and interval timing of sequential events are closely linked. We present a dynamic neural field model which exploits the existence and stability of multi-bump solutions with a gradient of activation to store serial order. The activation gradient is achieved by applying a state-dependent threshold accommodation process to the firing rate function. A field dynamics of lateral inhibition type is used in combination with a dynamics for the baseline activity to recall the sequence from memory. We show that depending on the time scale of the baseline dynamics the precise temporal structure of the original sequence may be retrieved or a proactive timing of events may be achieved.
\end{abstract}

Keywords: Dynamic Field Model, Serial Order, Interval Timing, Prefrontal Cortex.

\section{Introduction}

Virtually every aspect of our everyday routine actions is embedded in a sequential context. We see this in tasks like getting dressed, playing games, setting the dinner table or cooking a meal. The capacity to hold the temporal order of a short sequence of events in memory is of primary importance to our ability for efficient high-level planning when precise order of what has just happened and what is about to happen is of the essence. Very often a fluent and successful task execution requires not only a judgment about the ordinal sequence structure but also a metrical judgment that involves the analysis of elapsed time. Our ability to adjust behavior to temporal regularities in the environment in the range of seconds or minutes is called interval timing [1]. It manifests for instance when we start preparing an action in anticipation of a salient perpetual event that will happen in the nearer future.

Experimental evidence from physiological and behavioral studies suggests that the neural mechanisms supporting both ordinal and interval properties of time

* The work was supported by the PhD Grant SFRH/BD/41179/2007 from FCT.

T. Honkela et al. (Eds.): ICANN 2011, Part II, LNCS 6792, pp. 325-332, 2011.

(C) Springer-Verlag Berlin Heidelberg 2011 
are closely related (for discussion see e.g. [2/34]). It has been postulated that temporally predictable changes in self-sustained activity of prefrontal "delay" cells which are known for their role in working memory and action planning may be used by the brain to measure elapsed time [5].

In this paper we present a computational model based on dynamic neural fields 6] that aims at testing the idea of shared mechanisms for temporal and ordinal coding of sequential processes. More concretely, the model builds on three key neuroscientific findings. 1) Averbeck et al. 7] described firing patterns of cells in prefrontal cortex (PFC) in a sequential task that are consistent with the notion of a parallel processing of planned serial movements (see also [8]). They reported that prior to sequence onset neural population activity reflected each of all forthcoming goal-directed actions with a pre-activation gradient encoding serial order. 2) Various studies that directly investigated the neural basis of timing report ensemble activity showing a monotonic relationship between peak activity and elapsed time [39]. 3) Separate subpopulations in PFC represent intended future goal-directed actions and already accomplished goals [10].

Dynamic neural field models explain the capacity to hold an item of information "on-line" in short term memory as the result of strong recurrent interactions within neural populations that can sustain a persistent "bump" activity in the absence of external input 61116]. The model extends previous mathematical results on the existence of multiple bumps of equal strength [12 to implement a working memory of sequential events in which varying levels of self-sustained peak activity are correlated with the relative position of each item.

In what follows, we begin with a description of the model consisting of three coupled neural fields. We discuss mathematical details of the model in section 3 A report of a series of simulation results is given in section 4 followed by a discussion of results and related work.

\section{Model Description}

In the experiments designed to test the neural processing of serial order in goaldirected behavior typically simple reaching or saccadic eye movements towards objects are used. The temporal order may be defined by the spatial object position (that is, movement direction and amplitude) or object features like color, weight or size. The central idea of dynamic field models is that suprathreshold population activity of neurons tuned to these continuous dimensions represents specific parameter values. Fig. 1 presents an overview of the model architecture which is inspired by the experimental findings summarized in the Introduction. The self-sustained activation pattern in field $u_{S M}$ stores all items of a sequence with a strength of activation that decreases from item to item as a function of elapsed time since sequence onset. This activation gradient is achieved by combining a field dynamics that guarantees the evolution of a single, self-stabilized bump in response to a localized transient input representing a perceived event with a state-dependent threshold accommodation dynamics for the firing rate function [13. Through excitatory connections, neurons in $u_{S M}$ project their 
activation to corresponding neurons in field $u_{S R}$. This input leads to a subthreshold pre-activation of neural populations that mirrors the primacy gradient of strengths in $u_{S M}$. Sequence recall starts with a continuous increase of the baseline activity which brings neural populations closer to the threshold for the evolution of a self-stabilized bump. The order and timing of sequence elements can be retrieved by using the baseline dynamics to first trigger a suprathreshold response of the population with the highest pre-activation which then becomes suppressed due to inhibitory feedback from field $u_{P E}$. Self-stabilized population activity in this field is initially driven by the activation dynamics of the corresponding population in $u_{S R}$ and may thus be described as a memory for already achieved events or goals.

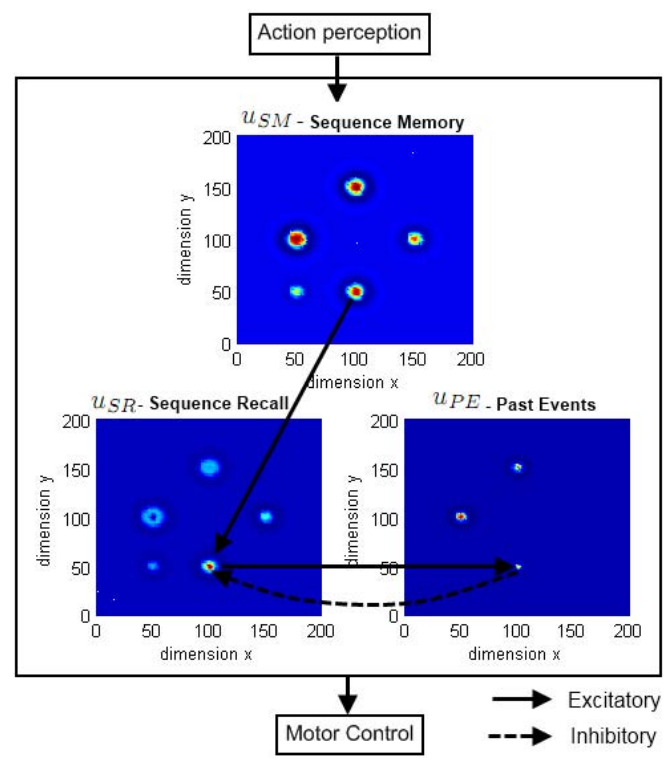

Fig. 1. The architecture of the field model

\section{Model Equations}

The dynamics in the sequence memory field is governed by the following equation [6]:

$\tau_{u_{S M}} \dot{u}_{S M}(x, t)=-u_{S M}(x, t)+h_{S M}(t)+\int w\left(x-x^{\prime}\right) f\left(u_{S M}\left(x^{\prime}, t\right)\right) d x^{\prime}+S(x, t)$

where $u_{S M}(x, t)$ represents the activity at time $t$ of a neuron encoding dimension $x$. The parameter $\tau_{u_{S M}}>0$ defines the time scale of the field. The firing rate function $f$ is taken as the Heaviside step function with threshold $0 . S(x, t)$ 
represents the time-dependent, localized input to the field. It is taken as a Gaussian centered at position $x$ with amplitude $S_{\text {strength }}>0$ and standard deviation $\sigma_{\text {input }}>0$. The connection function $w(x)$ determines the coupling between neurons within the field. It is well know that coupling function of lateral inhibition type used by Amari in his original work (1977) do not support stable multi-bump solutions 1216 . Using a coupling function with oscillatory rather than monotonic decay, Laing et al (2002) have shown that multiple regions of suprathreshold activity can persist in a field. To model working memory for several events separated in time we adapt the connection function $w$ used in [12]:

$$
w(x)=A e^{-b|x|}(b \sin |\alpha x|+\cos (\alpha x)),
$$

where the parameter $b>0$ controls the rate at which the oscillations in $w$ decay with distance. The parameters $A$ and $\alpha$ are added to control the amplitude and the zero crossings of $w$. The variable $h_{S M}(t)$ defines the baseline level of activation which we chose to vary with time. Note that by including $h_{S M}(t)$ in the definition of the firing rate function $f=f\left(u-h_{S M}(t)\right)$ it becomes clear that changing the baseline level is equivalent to changing the threshold of $f$. Following the idea of the phenomenological model for threshold accommodation in dynamic fields discussed by Coombes and Owen [13], we apply the following state-dependent dynamics for the baseline activity:

$$
\dot{h}_{S M}=\left(1-g\left(u_{S M}(x, t)\right)\right)\left(-h_{S M}+h_{S M_{0}}\right)+k g\left(u_{S M}(x, t)\right),
$$

where $g$ is chosen as the Heaviside step function, $h_{S M_{0}}$ defines the level to which $h_{S M}$ relaxes without suprathreshold activity at position $x$ and $k>0$ measures the growth rate when it is present. As the result of the baseline or threshold dynamics a primacy gradient of strengths is establish when at different points in time bumps evolve in response to external input. The standard deviation $\sigma_{\text {input }}$ is chosen to guarantee that the population evolves a single localized activity pattern centered at the stimulated field position. A minimum spatial distance between successive input presentations ensures that a multi-bump solution may stabilize.

The dynamics of the "sequence recall" field $u_{S R}$ and the "past events" field $u_{P E}$ are governed by the following equations, respectively:

$$
\begin{aligned}
\tau_{u_{S R}} \dot{u}_{S R}(x, t)= & -u_{S R}(x, t)+h_{S R}(t)+\int w_{S R}\left(x-x^{\prime}\right) f\left(u_{S R}\left(x^{\prime}, t\right)\right) d x^{\prime} \\
& -\int w\left(x-x^{\prime}\right) f\left(u_{P E}\left(x^{\prime}, t\right)\right) d x^{\prime}+u_{S M}(x), \\
\tau_{u_{P E}} \dot{u}_{P E}(x, t)= & -u_{P E}(x, t)+h_{P E}+u_{S R}(x, t) f\left(u_{S R}\left(x^{\prime}, t\right)\right) \\
& +\int w\left(x-x^{\prime}\right) f\left(u_{P E}\left(x^{\prime}, t\right)\right) d x^{\prime} .
\end{aligned}
$$

Since like $u_{S M}$ also $u_{P E}$ has to represent multi-bumps as stable solution we use the same connection function (2). The baseline activity $h_{P E}<0$ is constant. The situation is different for $u_{S R}$ where a single localized activity pattern represents 
a particular event during sequence recall. To ensure the existence of a 1-bump solution we use a connection function of lateral inhibition type given by:

$$
w_{S R}\left(x-x^{\prime}\right)=w_{\text {excite }} e^{\left(-\frac{\left(x-x^{\prime}\right)^{2}}{2 \sigma_{\text {excite }}^{2}}\right)}-w_{\text {inhib }} .
$$

The baseline activity $h_{S R}(t)$ evolves continuously in time described by the equation:

$$
\tau_{h_{S R}} \dot{h}_{S R}=1, \quad h_{S R}\left(t_{0}\right)=h_{u_{S R_{0}}}<0,
$$

where $\tau_{h_{S R}}$ controls the growth rate of $h_{S R}$.

\section{Simulations Results}

Fig. 2 shows a snapshot of a simulation of a one-dimensional version of the sequence model. To give a concrete example, the order of a sequence of goaldirected reaching movements may be defined by object color. There are 5 reaching movements to different objects that are successively executed. In the model, the series of localized inputs to the "sequence memory" field triggers a selfstabilized pattern consisting of 5 peaks (Fig 2, left). Due to the threshold accommodation dynamics (dashed line) the peak amplitudes reflect the temporal order of events. Fig 2 (right) shows the activation patterns in the "sequence recall" field (solid line) and the "past events" field (dashed line) at a time when all representations in $u_{S R}$ are below threshold because the activation peak at $x=100$ in $u_{P E}$ has just suppressed the representation of the first event and the representation of the second event at $x=60$ is just about to reach the threshold.
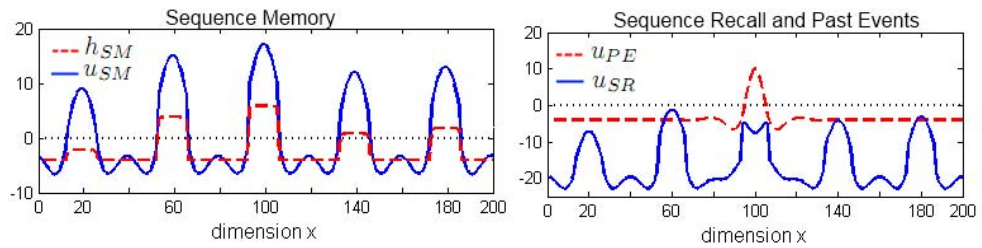

Fig. 2. A snapshot of a simulation of a one-dimensional version of the sequence model is shown. The following parameters value were used: For $u_{S M}, \tau_{u_{S M}}=20, h_{S M_{0}}=-4$, $k=0.01, S_{\text {strength }}=8, \sigma_{\text {input }}=4, x_{\text {input }} \in\{20,60,100,140,180\}$. For $u_{S R}, \tau_{u_{S R}}=20$, $\tau_{h_{S R}}=100, h_{S R_{0}}=-17, w_{\text {excite }}=16, \sigma_{\text {excite }}=4$ and $w_{\text {inhib }}=0.01$. For $u_{P E}$, $h_{P E}=-4$ and $\tau_{u_{P E}}=40$. The connection function parameters $b=0.15$ and $\alpha=0.3$ were equal for all fields, $A=2$ for $u_{S M}$ and $A=3$ for the others fields.

To directly compare the timing of events during encoding and recall, Fig. 3 compares the time courses of the population activity in the "sequence memory" field (top) and the "sequence recall" field (bottom) for two different choices of the time scale for the baseline dynamics in $u_{S R}$. Time $t=0$ indicates the start of the sequence and the first event (i.e., first object reached) is perceived at 
about $t=200$. Note that the events are irregularly spaced in time. As can be seen in the bottom figures, all subpopulations in the recall field appear to be from the beginning on pre-activated with a strength reflecting the rank order of execution. This model behavior reflects nicely the main findings about parallel processing of serial order in the neurophysiological study of Averbeck and colleagues (2002). If the time scale of the baseline dynamics is chosen as inversely related to the parameter $k$ controlling the growth rate of the threshold accommodation dynamics, $\tau_{h_{S R}}=1 / k$, the recall dynamics nearly perfectly reproduces the timing of events (bottom left). If the time scale for the baseline dynamics is chosen to be faster, $\tau_{h_{S R}}<1 / k$, proactive timing of events can be observed (bottom right). The total execution time decrease but the proportion of total time for each event of the sequence remains essentially invariant. It is beyond the scope of this article to compare model prediction directly with theoretical and experimental results about interval timing. It is however worth mentioning that the model behavior is in line with key principles [1]. When people are asked to speed up or slow down the execution of a movement sequence they do so with near constancy in the relative timing. Moreover, assuming that noise may affect the growth rate of the baseline shift from trial to trial the model predicts that the variability of time estimation grows proportionally with interval duration.
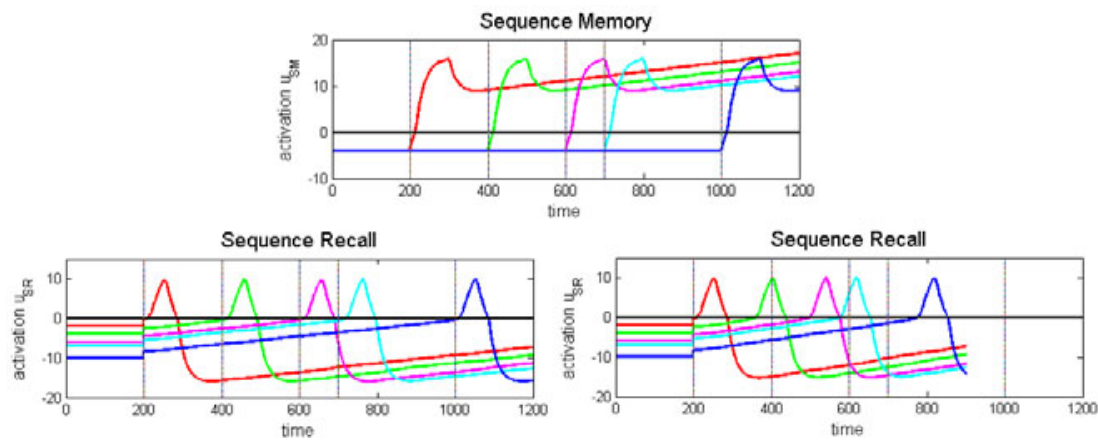

Fig. 3. The time course of the maximal activation of each subpopulation in Fig. 2(left) is shown for the sequence encoding (top) and the sequence recall (down) fields. For the recall $\tau_{h_{P E}}=100$ (left) and $\tau_{h_{P E}}=70$ (right) were chosen.
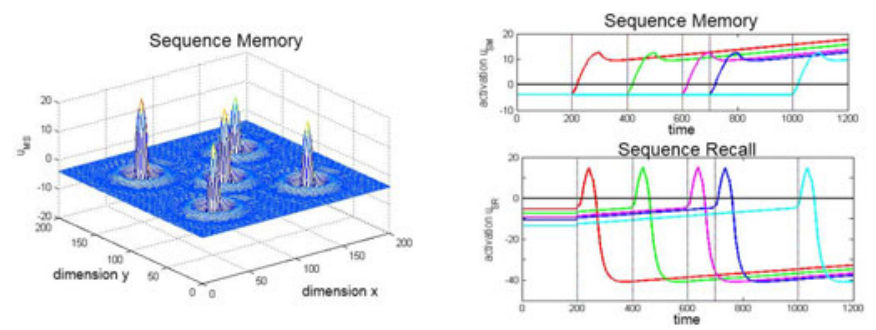

Fig. 4. Example of a model simulation in two dimensions. Self-stabilized bumps in $u_{S M}$ (left) and the time course of activity in $u_{S M}$ and $u_{S R}$ (right) are shown. 
It is straightforward to generalize the model to higher dimensions. Fig 4 shows an example of dynamic fields spanned over two dimensions representing for instance movement direction and amplitude. Stable multi-bump solutions in response to transient inputs exist for the two-dimensional analogue of the coupling function (2).

\section{Discussion}

We have presented a dynamic field model of sequential events that implements the idea of closely related neural systems for controlling the interval and ordinal dimensions. Serial order is stored in working memory by assuming that memory strength for each event decreases as a function of elapsed time between sequence onset and the event. During recall, the ordinal and temporal structure is recovered from the memory list by applying a simple dynamics for the baseline activity of the decision field. An interesting feature of the model is that a speeding up of the baseline dynamics leads to a proactive timing of events. For a cognitive agent such a mechanism may be important for instance to timely prepare the next action or to allocate attention. The field model shares key features like parallel response activation and activation gradient with "Competitive Cuing" models [14 15] that have been applied to a wide variety of serial order problems (mostly concerning the ordinal dimension). Compared to connectionist implementations, the dynamic field approach offers advantages because it allows us to rigorously understand the existence and stability of activation patterns and their dependence on external inputs 6 [ 12 13]. This understanding may guide the development of complex cognitive models.

One of our future goals is the validation of the sequence model as part of an existing dynamic field for human-robot interaction [17]. In the context of robotics applications it is important to stress that due to the self-stabilized properties of the field dynamics the model runs autonomously without feedback from the environment. This can be used by the robot for instance to "mentally" simulate the timing of sequential events. However, it is also possible and technically straightforward to include in the model sensory feedback as additional input necessary to trigger event representations in $u_{S R}$ or $u_{P E}$ [18.

In the present implementation the field model does not allow us to handle repeated items in a sequence. One possibility to overcome this limitation is to postulate the existence of a dynamic control signal that allows the activation gradient to vary during the course of sequence generation [15]. A neural substrate for such a control signal is not known and it would require an extra learning process to establish links between the signal and the sequence elements. For a dynamic field model a more parsimonious solution would be to add time as a continuous dimension along which items may be discriminated, just like perceptual or motor dimensions [19]. The existence of neural populations that are tuned to specific intervals of elapsed time might be interpreted as supporting this view 9 . 


\section{References}

1. Machado, A., Malheiro, M.T., Erlhagen, W.: Learning to Time: A Perspective. J. of the Experimental Analysis of Behavior 92, 423-458 (2009)

2. Dominey, P.F.: A shared system for learning serial and temporal structure of sensori-motor sequences? Evidence from simulation and human experiments. Cognitive Brain Research 6, 163-172 (1998)

3. Janssen, P., Shadlen, M.N.: A representation of the hazard rate of elapsed time in macaque area LIP. Nature Neuroscience 8(2), 234-241 (2005)

4. Staddon, J.E.R.: Interval timing: memory, not a clock. Trends in Cognitive Sciences 9, 312-314 (2005)

5. Lewis, P.A., Miall, R.C.: Remembering the time: a continuous clock. Trends in Cognitive Sciences 10, 401-406 (2006)

6. Amari, S.: Dynamics of pattern formation in lateral-inhibition type neural fields. Biological Cybernetics 27, 77-87 (1977)

7. Averbeck, B.B., Chafee, M.V., Crowe, D.A., Georgopoulos, A.P.: Parallel processing of serial movements in prefrontal cortex. Proc. Natl. Acad. Sci. 99, 13172-13177 (2002)

8. Tanji, J., Shima, K., Mushiake, H.: Concept-based behavioral planning and the lateral prefrontal cortex. Trends in Cognitive Sciences 11, 528-534 (2007)

9. Genovesio, A., Tsujimoto, S., Wise, S.P.: Neuronal activity related to elapsed time in prefrontal cortex. J. Neurophysiology 95, 3281-3285 (2006)

10. Genovesio, A., Brasted, P.J., Wise, S.P.: Representation of future and previous spatial goals by separate neural populations in prefrontal cortex. J. Neuroscience 26, 7305-7316 (2006)

11. Erlhagen, W., Schöner, G.: Dynamic field theory of motor preparation. Psychological Review 109, 545-572 (2002)

12. Laing, C.R., Troy, W.C., Gutkin, B., Ermentrout, G.B.: Multiple bumps in a neuronal model of working memory. SIAM J. on Applied Math. 63, 62-97 (2002)

13. Coombes, S., Owen, M.R.: Exotic dynamics in a firing rate model of neural tissue with threshold accommodation. AMS Cont. Math. 440, 123-144 (2007)

14. Grossberg, S.: Behavioral contrast in short term memory: Serial binary memory models or parallel continuous memory models. J. Math. Psych. 17, 199-219 (1978)

15. Houghton, G.: The problem of serial order: A neural network model of sequence learning and recall. In: Dale, R., Mellish, C., Zock, M. (eds.) Current Research in Natural Language Generation, pp. 287-319. Academic Press, London (1990)

16. Laing, C., Troy, W.: Two-bump solutions of Amari-type models of neuronal pattern formation. Physica D 178(3), 190-218 (2003)

17. Bicho, E., Louro, L., Erlhagen, W.: Integrating verbal and non-verbal communication in a dynamic neural field architecture for human-robot interaction. Front. Neurorobot. 4, 5 (2010), doi:10.3389/fnbot.2010.00005

18. Sandamirskaya, Y., Schöner, G.: An embodied account of serial order: How instabilities drive sequence generation. Neural Networks 23(10), 1164-1179 (2010)

19. Farrell, S., McLaughlin, K.: Short-term recognition memory for serial order and timing. Memory \& Cognition 35, 1724-1734 (2007) 